\title{
Communication
}

\section{Perfil de tutores de cães e gatos sobre as zoonoses no município de Patos de Minas - MG}

Received: oct, 2018; Accepted: feb, 2020

\author{
Iara Maria Alves Rodrigues ${ }^{*}$, Denis Prudencio Luiz², \\ Guilherme Nascimento Cunha ${ }^{3}$
}

\begin{abstract}
Resumo
$\mathrm{O}$ vínculo afetivo entre o homem e os animais está cada vez mais forte. Porém, os cães e os gatos exigem certos cuidados, que quando não são praticados acarretam problemas à saúde pública. $\mathrm{O}$ estudo teve como objetivo avaliar o perfil de tutores de cães e de gatos acerca das zoonoses no município de Patos de Minas-MG. Esta pesquisa foi realizada por meio de um questionário aplicado em forma de entrevista no período de julho a agosto de 2016. Ao todo 300 tutores foram entrevistados, de forma individual. O trabalho foi realizado em seis bairros distintos e de diferentes classes sociais da cidade. Para o tratamento dos dados coletados foi utilizada análise estatística descritiva com o auxílio do software BioEstat versão 5.3. Sobre zoonoses, 78,66\% (236/300) dos tutores relataram ter conhecimento de que os animais podem transmitir doenças ao homem, sendo raiva a mais citada, com 33\% (184/300). No que diz respeito como as zoonoses podem ser transmitidas ao homem, por volta de $13 \%$ (39/300) não souberam responder. Concluiu-se que, apesar da população entender que os animais podem transmitir doenças ao homem, muitas pessoas carecem de conhecimentos relevantes, refletindo a carência de informações que lhes é fornecida. Evidencia-se então, o desafio para os órgãos públicos responsáveis e aos médicos veterinários, no sentido de informar e educar os tutores quanto às zoonoses e as suas reais implicações.

Palavras-chave: Saúde Pública, Zoonoses, Questionário, Animais de Companhia, Canino, Felino.
\end{abstract}

\footnotetext{
1. Curso de Medicina Veterinária - Centro Universitário de Patos de Minas - Unipam, Patos de Minas, MG, Brasil.

${ }^{2}$ Laboratório de Patologia Molecular e Biotecnologia - PMBIO, Universidade Federal de Uberlândia, Uberlândia, MG, Brasil.

${ }^{3}$ Curso de Medicina Veterinária - Centro Universitário de Patos de Minas - Unipam, Patos de Minas, MG, Brasil.

* Corresponding author: R. Frei Patrício, nº 590, Centro - João Pinheiro/MG, (38) 99214-5005. E-mail: iaraalvesvet@hotmail.com,
} 


\section{Introdução}

Com as mudanças nos hábitos sociais e culturais se criou um vínculo, muitas vezes familiar, entre os homens e os animais. Entretanto, essa ligação requer inúmeros cuidados, que se não exercidos geram uma série de consequências para ambas as espécies, destacando-se a proliferação de zoonoses (LANGONI et al., 2011).

Nesse sentido a conscientização dos tutores em relação ao abandono é de suma importância, uma vez que esse ato gera aumento na população de animais que já se encontram nas ruas (FERREIRA, 2010). Esse fato constitui agravos na saúde pública devido à grande variedade de microganismos transportados por cães e gatos e à estreita relação destes com a espécie humana, já que a transmissão de numerosas doenças se torna propensa (REASER; CLARK; MEYERS, 2008).

Sendo assim, pode-se entender as zoonoses como enfermidades transmissíveis de forma natural de animais vertebrados ao homem e viceversa, representando uma ameaça considerável à saúde pública. Ao longo dos anos, mais de 200 tipos de doenças foram descritos em vários países, sendo que nos últimos dez anos, surgiram mais de $75 \%$ de novas afecções em humanos com origem em agentes patogênicos provenientes de animais ou de produtos de origem animal (OMS, 2011).

Pelo menos $61 \%$ de todos os patógenos humanos são zoonóticos, e representaram $75 \%$ de todos os patógenos emergentes durante a última década (OMS, 2011). A transmissão das zoonoses para o homem, geralmente, ocorre diretamente pelo contato com animais infectados, os quais eliminam o agente pelas secreções; e, indiretamente, pela ingestão de animais ou pela água contaminada. A participação de vetores e a interação com o ambiente também pode ocorrer (LANGONI, 2004).

Dentre as várias doenças de caráter zoonótico têm-se, por exemplo: raiva, leptospirose, toxoplasmose, leishmaniose cutânea e visceral, dentre 
outras (LANGONI, 2004). Também é relevante considerar as zoonoses que são transmitidas através de vetores como pulgas e carapatos, e as dermatoses de cães e gatos sendo responsáveis por causas importantes de doenças cutâneas no homem, posto que aproximadamente $5 \%$ dos casos dermatológicos nessa espécie são atribuídos diretamente aos ectoparasitas de animais (SCOTT; HORN, 1987).

Portanto, é necessária a conscientização dos cidadãos, bem como a disseminação de informações sobre conceitos básicos referente à prevenção de zoonoses, pois conforme Langoni et al. (2011) a população reconhece a importância dela, mas não a exerce em sua totalidade.

O presente estudo teve como objetivo determinar o conhecimento dos proprietários de cães e gatos sobre os princípios que regem a zoonose bem como suas implicações na saúde pública.

\section{Material e métodos}

O presente estudo foi realizado com tutores de cães e gatos no Município de Patos de Minas - MG diante do acompanhamento domiciliar de agentes de saúde municipal, no período da manhã, de julho a agosto de 2016, sendo aprovada pelo Comitê de Ética no Uso de Animais (Ceua) do Centro Universitário de Patos de Minas - Unipam, sob o protocolo n²46/16.

O município de Patos de Minas está situado na região intermediária às regiões do Triângulo Mineiro e do Alto Paranaíba, MG, Brasil. Com um PIB per capta de $\mathrm{R} \$ 23.834,98$, Patos de Minas possui um Índice de Desenvolvimento Humano Municipal (IDHM) de 0,765 e uma área territorial de $3.190,187 \mathrm{~km}^{2}$. Sua população conta com 91,6\% dos domicílios com esgotamento sanitário adequado e uma média salarial dos trabalhadores formais de 2,2 salários mínimos, com um índice Gini de 0,41 apresentando, assim, uma menor desigualdade na população (IBGE, 2016). Na presente pesquisa foram avaliados proprietários de cães e gatos domiciliados de ambos 
os sexos e de diferentes raças e idades, sendo considerada a presença de apenas um animal por residência.

A estimativa populacional para 2017 é de 150.893 habitantes (IBGE, 2016). Como fim de alcançar diferentes perfis de resposta foram selecionados, previamente, seis bairros, sendo igualmente divididos em classe baixa, classe média e classe média alta. Para o cálculo da proporção animais de estimação/homem foi utilização a relação de 1:6 (MASCOLLI et al., 2002), resultando em um total de 25.148 animais. Com a finalidade de calcular a amostra, considerou-se um nível de confiança de $95 \%$ e uma variação de $5 \%$. Levantamentos recentes demonstram que 66,7\% dos donos de pet preferem a criação de cães, enquanto 19,3\% a criação de gatos (CARDOSO et al., 2016). As fórmulas utilizadas foram (THRUSFIELD, 2004):

$\mathrm{n}=1,96^{2} \mathrm{P}_{\text {esp }}\left(1-\mathrm{P}_{\text {esp }}\right) / \mathrm{d}^{2} \mathrm{n}_{\text {cor }}=(\mathrm{N} \times \mathrm{n}) /(\mathrm{N}+\mathrm{n})$

Sendo:

$\mathrm{n}$ = tamanho da amostra baseada em população infinita

$\mathrm{P}_{\mathrm{esp}}=$ prevalência esperada de cães ou gatos

$\mathrm{d}=$ precisão absoluta desejada

$\mathrm{n}_{\mathrm{cor}}=$ tamanho da amostra examinada

$\mathrm{N}=$ tamanho da população em estudo

Substituindo os valores para cães:

$\mathrm{n}=1,96^{2} \times 0,67(1-0,67) / 0,05^{2}$

$\mathrm{n}=3,86 \times 0,2211 / 0,0025$

$\mathrm{n}=341,4$

$\mathrm{n}_{\mathrm{cor}}=(25148 \times 341,4) /(25148+341,4)$

$\mathrm{n}_{\text {cor }}=8585527 / 25489,4$

$\mathrm{n}_{\mathrm{cor}}=337$

Substituindo os valores para gatos:

$\mathrm{n}=1,96^{2} \times 0,19(1-0,19) / 0,05^{2}$

$\mathrm{n}=3,86 \times 0,1539 / 0,0025$ 


$$
\begin{aligned}
& \mathrm{n}=237,6 \\
& \mathrm{n}_{\text {cor }}=(25148 \times 237,6) /(25148+237,6) \\
& \mathrm{n}_{\text {cor }}=5975164,8 / 25385,6 \\
& \mathrm{n}_{\text {cor }}=235
\end{aligned}
$$

Resultando um ncor médio de 286

Embora a amostra tenha sido de 286 proprietários, decidiu-se por aumentar o número de questionários para 300 no intuito de trabalhar com uma melhor base de cálculos.

Assim, foram aplicados questionários in loco na residência de 300 tutores de cães e gatos. Durante o processo de entrevista foi entregue aos proprietários uma via do Termo de Consentimento Livre e Esclarecido referente à pesquisa.

Como instrumento para esta pesquisa foi utilizado o questionário semiestruturado sobre zoonoses, sendo constituído de perguntas fechadas de múltipla escolha referentes ao: a) tutor - nível de escolaridade e faixa etária b) animal - espécie, raça e se o pet é castrado ou não e c) questões específicas do tema zoonoses: o animal tem acesso à rua?; Se o proprietário sabe que os animais podem transmitir doenças para o homem?; Se ele conhece alguma dessas doenças?; Se possui conhecimento sobre como essas doenças podem ser transmitidas dos animais para o homem? Com o objetivo de padronizar as entrevistas e evitar vieses, foi realizado um piloto a fim de adequar o questionário.

Para o tratamento dos dados coletados foi utilizada análise estatística descritiva com o auxílio do software BioEstat versão 5.3. 


\section{Resultados e discussão}

Todos os responsáveis inqueridos (100\%) aderiram ao estudo e responderam de forma satisfatória o questionário.

Quanto ao perfil dos tutores observou-se que a faixa etária predominante foi a dos tutores adultos, correspondendo a 53\% (159/300) dos entrevistados conforme a Tabela 1. Esse mesmo resultado também foi observado em estudo feito por Tome et al. (2010), em que foi possível observar que a maioria dos entrevistados $(71,77 \%)$ compreendiam idades entre 21 e 60 anos. Esse resultado pode estar relacionado ao horário em que foi feita a pesquisa, manhã e tarde, sendo que nesses turnos espera-se que as crianças e os jovens estejam frequentando as escolas.

Tabela 1. Frequência absoluta e relativa do perfil dos tutores avaliados pelo questionário, Patos de Minas, MG, 2016

\begin{tabular}{cccc}
\hline Informações do tutor & Opções de resposta & $\mathrm{N}$ & $\%$ \\
\hline Faixa etária & Criança & 2 & 0,66 \\
& Adolescente & 15 & 5,00 \\
& Adulto Jovem & 77 & 25,66 \\
& Adulto & 159 & 53,00 \\
& Idoso & 47 & 15,66 \\
& Nenhum & 3 & 1,00 \\
& Ensinol de escolaridade & 47 & 15,66 \\
& Ensino Médio & 157 & 52,33 \\
& Graduado & 93 & 31,00 \\
\hline
\end{tabular}

Referente à escolaridade, a maioria dos entrevistados, 52,33\% (157/300), possuíam o Ensino Médio completo (Tabela 1). Porcentual inferior foi encontrado em um estudo feito por Lima et al. (2010) na cidade de Recife (PE), onde foi entregue a alunos do maternal, jardim II e alfabetização de uma escola particular e da pré-escola, um questionário para que seus pais respondessem. Percebeu-se que a apenas 32,8\% (21/64) dos participantes tinham concluído o Ensino Médio, refletindo que a população tem buscado a continuidade escolar sendo que o acesso às instituições de ensino está mais 
facilitado, através de programas do Ministério da Educação, como a Educação de Jovens e Adultos (EJA).

No que tange à espécie escolhida pelos tutores, 81\% (243/300) foi a canina, seguido de 19\% (57/300) pela felina, conforme Tabela 2. Esse resultado pode estar relacionado com o comportamento dos cães, uma vez que estes normalmente são mais alegres, expressam mais carinho e mantêm um maior contato corporal com seus tutores sendo, no entanto, mais dependentes deles. Já os gatos mostram-se mais independentes e isolados em relação ao proprietário passando uma percepção equivocada de indiferença em relação a ele (CATAPAN et al., 2015).

Foi possível detectar que apenas 16,66\% (50/300) dos animais eram esterilizados (Tabela 2). Destes, 62\% (31/50) eram machos. Esse achado reflete ao fato de os tutores terem conhecimento de que os animais desse gênero, quando castrados, ficam menos territorialistas e ariscos, e mais caseiros. Dados estes também corroborados por Heidenberger e Unshelm (1990) e por Oliveira (2002).

Quando se avaliou o padrão racial (Tabela 2) a maior parte, 57\% (171/300), era sem raça definida (SRD), sendo que destes, 21,63\% (37/171) eram esterilizados. Já em relação aos com raça definida (CRD), 43\% (129/300) eram de diversas raças, com apenas 10,07\% (13/129) destes castrados. A baixa frequência de animais CRD esterilizados reflete uma pretensão dos tutores em ter acesso aos filhotes e, com isso, ter um certo lucro. Porém, esse hábito tem impactos negativos na saúde pública, uma vez que quanto maior o número de fêmeas de raça se reproduzindo menor será a oportunidade de filhotes SRD serem adotados, o que consequentemente aumenta o número de cães não domiciliados e, assim, maior serão as chances de propagação de zoonoses, agravando a saúde pública. 
Tabela 2. Frequência absoluta e relativa do perfil dos animais avaliado pelo questionário, Patos de Minas, MG, 2016.

\begin{tabular}{cccc}
\hline Perfil dos Animais & Opções de resposta & $\mathrm{N}$ & $\%$ \\
\hline \multirow{2}{*}{ Espécie } & Cão & 243 & 81,00 \\
& Gato & 57 & 19,00 \\
Raça & SRD* & 171 & 57,00 \\
& CRD* & 129 & 43,00 \\
Castrado & Sim & 50 & 16,66 \\
& Não & 250 & 83,33 \\
\hline
\end{tabular}

*SRD: sem raça definida; *CRD: com raça definida.

Sobre a questão referente ao acesso do animal à rua, pouco mais da metade dos entrevistados, 52,33\% (157/300), relatou que seus animais passeiam apenas acompanhados e na coleira. Esse resultado transmite a imprudência da metade da população $(47,67 \%)$ em deixar seus animais livres pelas vias públicas. Infelizmente, é comum o hábito dos tutores soltarem os seus animais para que estes realizem passeios livremente ou, muitas vezes, para que estes defequem fora de seus quintais, porém esse ato causa vários problemas à sociedade, tais como produção de dejetos e dispersão de resíduos; acidentes de trânsito; agressões, tanto a outros animais quanto às pessoas, $\mathrm{e}$ acasalamentos, que aumentam a população canina (LANGONI et al., 2011).

Sobre o conceito do significado do termo zoonoses, 78,66\% (236/300) dos tutores relataram ter conhecimento de que os animais podem transmitir doenças ao homem. Porém 21,33\% (64/300) não conhece nenhuma dessas doenças que podem ser carreadas à população humana, refletindo a carência de informação que a população possui. Assim, em concordância com Junior (2012), fica claro a importância do Médico Veterinário no Núcleo de Apoio a Saúde da Família (NASF), que com as competências próprias da profissão, será criado um elo entre a saúde animal e humana. 
Quando solicitados a darem exemplos sobre as doenças consideradas como zoonoses, a raiva foi a doença mais citada no presente inquérito, com 61,33\% (184/300) dos entrevistados. Esse fato deve-se às ações de educação em saúde voltada para tal enfermidade, como campanhas de vacinação de âmbito nacional realizadas todo ano por cada município. A leishmaniose foi mencionada por 48,66\% (146/300) dos tutores e 37\% (111/300) citaram sarna. Já a toxoplasmose foi lembrada em 21\% (63/300) das vezes, seguida por leptospirose com 15,66\% (47/300).

Figura 1. Relação de zoonoses mencionadas pelos tutores, Patos de Minas, MG, 2016

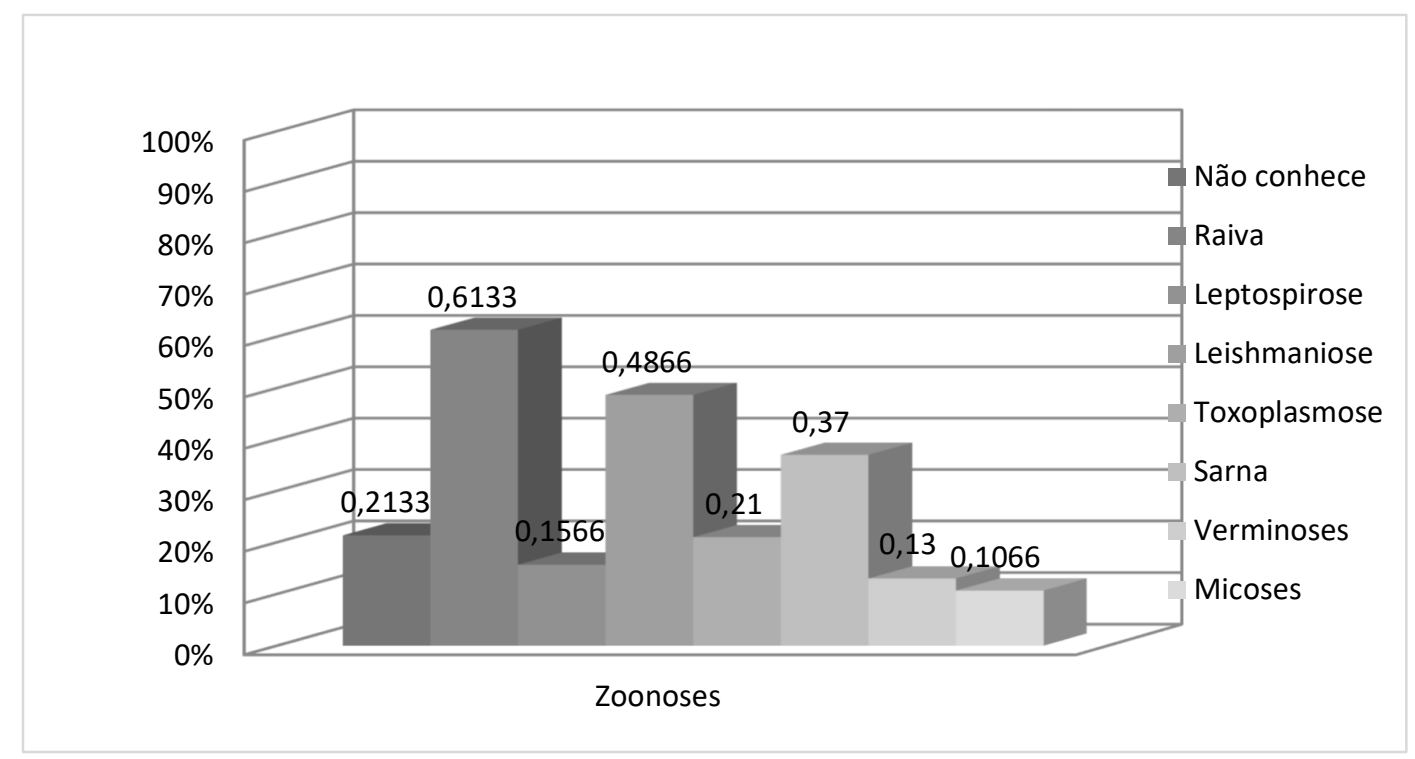

As verminoses com 13\% (39/300), e, por último, as micoses perfazendo 10,66\% (32/300) (Gráfico 4). Resultados semelhantes foram encontrados em estudos feitos por Catapan et al. (2015) e por Lima et al. (2010), em que a raiva foi a doença mais citada. Já Sampaio (2014) obteve resultado distinto, no qual a leptospirose foi à doença mais mencionada, seguida, então, da raiva.

Os tutores foram interrogados ainda como as zoonoses podem ser transmitidas ao homem, do qual cerca de 13\% (39/300) não souberam responder e 87\% (261/300) citaram alguma forma de transmissão, sendo que destes, $62,66 \%$ (188/300) mencionaram a mordedura. O contato direto (ato de 
pegar, abraçar e beijar o animal) foi lembrado por 54\% (162/300) dos entrevistados, enquanto o contato indireto (contato com fômites) por 38,33\% (115/300), seguido por fezes em 29,66\% (89/300), urina 21\% (63/300), saliva $13,33 \%$ (40/300), sangue 7\% (21/300), arranhadura 6\% (18/300) e ingestão de alimentos com 2,33\%(7/300). Conforme o Gráfico 5.

Figura 2. Relação da forma de transmissão de zoonoses mencionada pelos tutores, Patos de Minas, MG, 2016

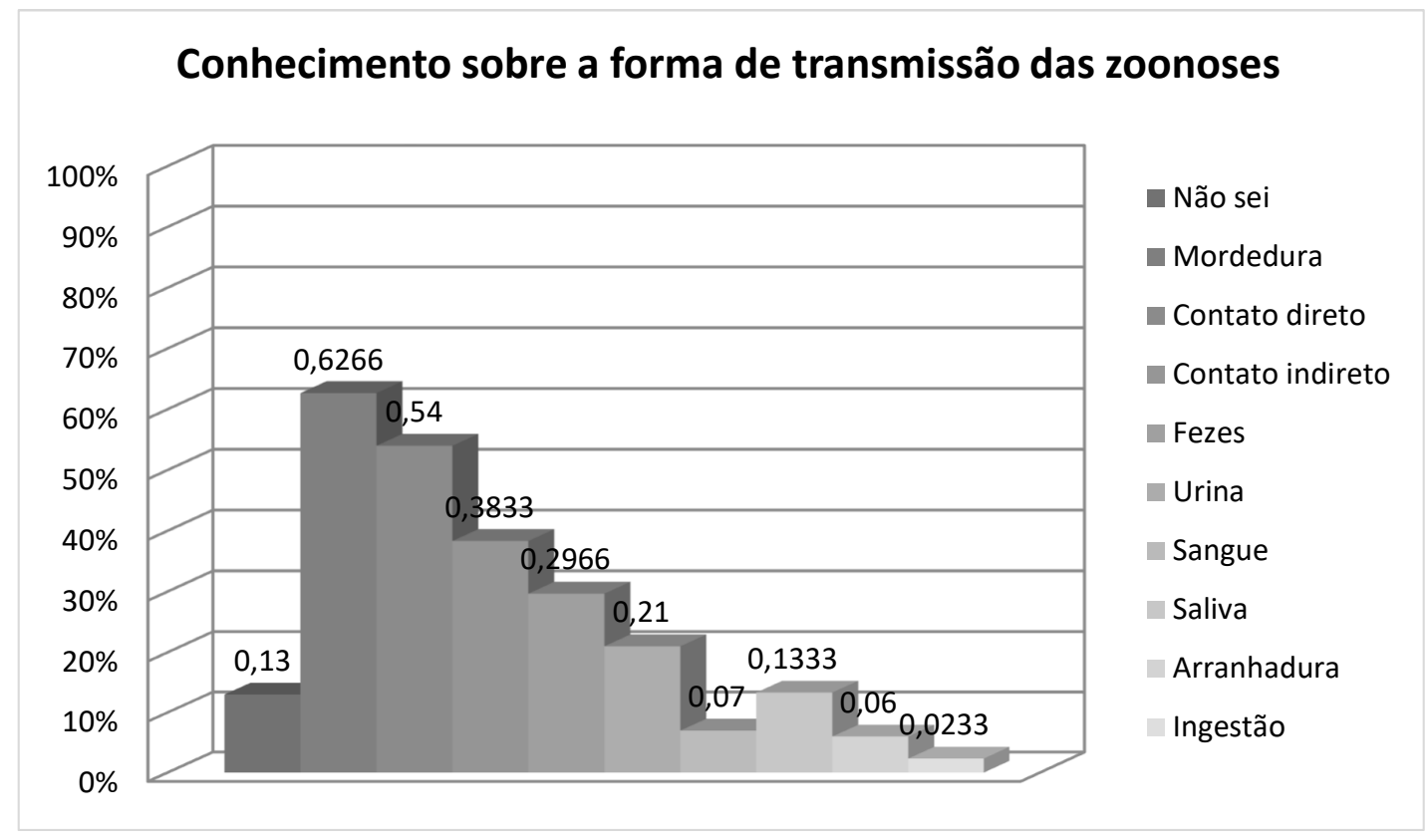

O meio de transmissão de zoonoses através da mordedura foi citado com maior frequência, sendo em grande parte das vezes relacionada à raiva, que, como anteriormente descrito, deve-se a campanha de vacinação de cães e gatos realizada anualmente no município em questão. $\mathrm{O}$ contato direto e indireto foram o segundo e o terceiro item mais citado, respectivamente. Resultados estes, semelhantes aos do estudo feito por Sampaio (2014). Essa percepção pelos tutores deve-se provavelmente devido ao contato mais estreito que há atualmente entre eles e seus animais de estimação e à conscientização de que animais transmitem doenças aos seres humanos e vice-versa. 
A grande lacuna de conhecimento da população relacionado às zoonoses observada neste estudo mostra-se como um indicador da necessidade de investimentos em campanhas nacionais e/ou municipais pelo Estado, tendo sua divulgação realizada por diferentes mídias. Uma vez que a prevenção de zoonoses começa pela conscientização da população, que é obtida com práticas de educação através de profissionais da saúde. O grande exemplo é a raiva, que é quase totalmente prevenida por meio da educação continuada (RUPPRECHT; HANLON; HEMACHUDHA, 2002).

\title{
Conclusão
}

Pôde-se concluir que a população apresentou ter conhecimento de que os animais podem transmitir doenças ao homem, porém mostra-se ainda carente de informações sobre quais são essas zoonoses e como podem ser transmitidas. Esses resultados evidenciam a importância da educação em saúde pública para a população e um desafio ao Estado para promover campanhas de conscientização.

\section{$* * *$ \\ Profile of tutors of dogs and cats on zoonoses in of Patos de Minas city}

\begin{abstract}
The affective bond between man and animals is growing stronger. However, dogs and cats require certain care, which when not practiced entail problems for public health. The objective of this study was to evaluate the profile of dog and cat tutors on zoonoses in the municipality of Patos de Minas-MG. For this, a questionnaire was applied in the form of an interview in the period from July to August 2016. In all, 300 tutors were interviewed individually. The work was carried out in six different neighborhoods and different social classes of the city. For the treatment of the collected data, a descriptive statistical analysis was used with the aid of BioEstat software version 5.3. On zoonoses, $78.66 \%$ (236/300) of the tutors reported having knowledge that the animals can transmit diseases to the man, being most cited anger with $33 \%$ (184/300). As far as zoonoses can be transmitted to humans, around $13 \%$ (39/300) did not know how to respond. It was concluded that although the
\end{abstract}


population understands that animals can transmit diseases to humans, many people lack relevant knowledge, reflecting the lack of information that is provided to them. The challenge for the responsible public agencies and the Veterinary Doctors is therefore to inform and educate tutors about zoonoses and their real implications.

Keywords: Public Health, Zoonoses, Questionnaire, Companion Animals, Canine, Feline.

\section{Referências}

CARDOSO, D. P; OLIVEIRA, R. P; ESTRELA, D. S; SARAIVA, L. A;FARIAS, M. P. O;SILVA, P. O. Perfil dos tutores de cão e gato no município de Bom Jesus-PI. PUBVET, v.10, n.8, p.580-586, 2016. https://doi.org/10.22256/pubvet.v10n8.580-586

CATAPAN, D.C. JUNIOR, J. A. V; WEBER, S. H; MANGRICH, R. M. V; SZCZYPKOVSKI, A. D; CATAPAN, A; PIMPÃO, C. T. Percepção e atitudes do ser humano sobre guarda responsável, zoonoses, controle populacional e cães em vias públicas. Revista Brasileira de Ciência Veterinária, v. 22, n. 2, p. 92-98, 2015. https://doi.org/10.4322/rbcv.2015.358

FERREIRA, K. Bem-estar animal e o trabalho do Centro de controle de zoonoses de Ponte Nova. Monografia. UNIVIÇOSA. 2010.

HEIDENBERGER; UNSHELM. Changes in the behavior of dogs after castration. TierarztlPrax. v.18, p 69-75, 1990.

IBGE - Instituto Brasileiro de Geografia e estatística. Censo Demográfico, 2010. Disponível em: http://cidades.ibge.gov.br/xtras/temas.php?lang=\&codmun=314800\&idtema=1\&sea

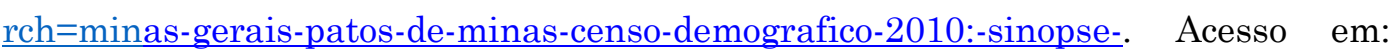
04/03/17. 
IBGE - Instituto Brasileiro de Geografia e Estatística. Censo Demográfico, 2016. Disponível em: https://cidades.ibge.gov.br/brasil/mg/patos-de-minas/panorama. Acesso em: 02/03/2017.

JUNIOR, A.M.F; FONSECA, A.G. A inclusão do Médico Veterinário na atenção básica à saúde da família. VII CONNEPI. Tocantins. 2012.

LANGONI, H.; TRONCARELLI, M.Z.; RODRIGUES, E.C.; NUNES, H.R.C.; HARUMI, V.; HENRIQUES, M.V.; SILVA, K.M.; SHIMONO, J. Conhecimento da população de Botucatu-SP sobre guarda responsável de cães e gatos. Veterinária e Zootecnia, v. 18, n. 2, p. 297-305, 2011.

LANGONI, H. Zoonoses and human beings. J. Venom. Anim. Toxinsincl. Trop. Dis [online]. 2004, vol.10, n.2 [cited 2009-09-21], pp. 111-111. Disponível em: http://www.scielo.br/scielo.php?script=sci_arttext\&pid=S1678-91992004000200001 ISSN 1678-9199. http://doi.org/10.1590/S1678-91992004000200001 Acesso em: $19 / 05 / 16$.

LIMA, A; ALVES, C. L; FAUSTINO, M. A. D. G; LIRA, N. M. S. D. Percepção sobre o conhecimento e profilaxia das zoonoses e posse responsável em pais de alunos do pré-escolar de escolas situadas na comunidade localizada no bairro de Dois Irmãos na cidade do Recife (PE). Ciênc. saúde coletiva [online]. 2010, vol.15, suppl.1, pp.1457-1464. ISSN 1413-8123. Disponível em: http://dx.doi.org/10.1590/S141381232010000700057. Acesso em 12/04/16 https://doi.org/10.1590/S1413$\underline{81232010000700057}$

MASCOLLI, R; PINHEIRO, S.R; VASCONCELLOS, S.A; FERREIRA, F; MORAIS, Z.M; PINTO, C.O; SUCUPIRA, M.C.A; DIAS, R.A; MIRAGLIA, F; CORTEZ, A; SILVEIRA DA COSTA, S; TABATA, R; MARCONDES, A.G. Inquérito sorológico para leptospirose em cães do município de Santana de Parnaíba, São Paulo, utilizando a campanha de vacinação anti-rábica do Ano de 1999. Arq. Inst. Biol., São Paulo, v.69, n.2, p.25-32, abr./jun., 2002 
OLIVEIRA, A.P.F. Comportamento social de machos e fêmeas castrados do gato doméstico (Feliscatus L.) em confinamento, 2002. Dissertação (Mestrado em Medicina Veterinária), Universidade de São Paulo, Ribeirão Preto-SP, 2002.

ORGANIZAÇÃO MUNDIAL DE SAÚDE - OMS (2011). Zoonoses. Disponível em: http://www.who.int/topics/zoonoses/en/. Acesso em 30/08/2016.

REASER, J. K; CLARK, E. E. JR; MEYERS, N. M. All Creatures Great and Minute: A Public Policy Primer for Companion Animal Zoonoses. ZoonosesPublicHealth 2008; v.55, n. 8-10, p. 385-401.

RUPPRECHT, C. E; HANLON, C. A; HEMACHUDHA, T. Rabies re-examined. The Lancet Infectious Diseases, New York, 2 (6): 327-343, 2002. https://doi.org/10.1016/S1473-3099(02)00287-6

SAMPAIO, A. B. Percepção da população do município de Cruz Alta (RS) sobre zoonoses transmitidas por cães e gatos. Acta Veterinaria Brasilica, v. 8, n. 3, p. 179$185,2014$.

SCOTT, D. W.; HORN Jr, R. T. Zoonotic dermatoses of dogs and cats. Veterinary Clinics of North America. Small Animal Practice, v.17, n. 1, p.117-120, 1987. https://doi.org/10.1016/S0195-5616(87)50609-X

THRUSFIELD, M. Epidemiologia Veterinária. 2. ed. São Paulo: Roca, 2004, 556p.

TOME, R. O; LANGONI, H; PERUCA, L. C. B; BABBONI, S. L. Avaliação do Conhecimento Sobre Algumas Zoonoses com Proprietários de Cães da Área Urbana do Município de Botucatu-SP. UNOPAR Científica. Ciências Biológicas e da Saúde. V.12, n.3. p.67-74, 2010. 